\title{
Leptomycin B induces apoptosis in cells containing the whole HPV 16 genome
}

\author{
CAROL E. JOLLY ${ }^{1}$, LINDSEY J. GRAY ${ }^{1}$, JOANNA L. PARISH ${ }^{1}$, SONIA LAIN ${ }^{2}$ and C. SIMON HERRINGTON ${ }^{1}$ \\ ${ }^{1}$ Bute Medical School, University of St Andrews, Bute Medical Buildings, Westburn Lane, \\ St Andrews, Fife, KY16 9TS, UK; ${ }^{2}$ Department of Surgery and Molecular Oncology, \\ Ninewells Hospital and Medical School, University of Dundee, Dundee, DD1 9SY, UK
}

Received April 9, 2009; Accepted June 5, 2009

DOI: 10.3892/ijo_00000377

\begin{abstract}
Cervical cancer is a major cause of death in women worldwide and is strongly associated with human papillomavirus (HPV) infection. Integration of HPV is thought to be a key step in malignant progression, and is associated with loss of regulation of the viral E6 and E7 oncogenes. Leptomycin B (LMB), a nuclear export inhibitor, has previously been shown to induce apoptosis in primary keratinocytes transduced with the HPV 16 E7 or E6/E7 genes, but not in normal cells. We show here that LMB can also induce apoptosis in derivatives of the W12 cell line that contain either episomal or integrated HPV 16. Cells transduced with HPV 16 E7 or E6/E7, and the episomal and integrated W12 derivatives showed distinct temporal expression patterns of the apoptotic markers activated caspase- 3 and M30. The expression of both markers occurred later in the episomal derivatives than in either transduced cells or W12 derivatives containing integrated HPV. These findings suggest that, although LMB can induce apoptosis in keratinocytes containing episomal or integrated HPV 16, genome status is likely to influence the response of HPV-associated anogenital lesions to LMB treatment.
\end{abstract}

\section{Introduction}

Human papillomaviruses (HPVs) are aetiologically linked to both benign lesions and neoplasia. These small, epitheliotropic,

Correspondence to: Professor C. Simon Herrington, Bute Medical School, University of St Andrews, Bute Medical Buildings, Westburn Lane, St Andrews, Fife KY16 9TS, UK

E-mail: csh2@st-andrews.ac.uk

Abbreviations: HPV, human papillomavirus; LMB, leptomycin B; NES, nuclear export signal; SIL, squamous intraepithelial lesion; PHK, primary human keratinocyte; HRP, horseradish peroxidase; act-casp-3, activated caspase 3; CK, cytokeratin; AU, arbitrary units

Key words: human papillomavirus, W12 derivatives, integration, leptomycin B, apoptosis double-stranded DNA viruses contain a circular $8 \mathrm{~kb}$ genome encoding only eight genes, which are subdivided into early (E1, E2, E4, E5, E6 and E7) and late (L1 and L2) genes (reviewed in ref. 1). The varying ability of the different HPV types to induce either benign lesions or anogenital neoplasia has led to their classification as either high risk (e.g. HPV 16 or 18) or low risk (e.g. HPV 6 or 11) types (2).

We have previously shown that the nuclear export inhibitor LMB can induce apoptosis in primary human keratinocytes (PHKs) expressing HPV 16 E7 and E6/E7 (3), but it is not known whether this effect also occurs in non-malignant cells that contain the whole viral genome and express these genes in a more natural context. Similarly, it is not known if integration of HPV DNA into the host genome, an important step in viral carcinogenesis, influences this effect. In a normal HPV infection the viral genome is found as circular episomes within the cell $(4,5)$ and gene expression remains under normal regulatory control $(6,7)$. Upon integration, the viral genome breaks and inserts into the host DNA. While the site of integration appears to be random, the site of viral genome disruption is predominantly confined to the viral regulatory E1/E2 region (8), leading to loss of these gene functions $(7,9)$ and consequent upregulation of E6 and E7 which normally target cellular p53 and pRb, respectively.

LMB, also known as elactocin, inhibits nuclear export at low $\mathrm{nM}$ concentrations by inhibiting the nuclear export signal (NES) receptor CRM1, a component of the nuclear pore (10). LMB prevents the nuclear export of proteins utilising the REV type NES signal, but does not appear to affect nuclear import (10). In our previous study (3), LMB induced apoptosis in keratinocytes expressing HPV 16 E7 and E6/E7 but not in normal keratinocytes, suggesting that LMB may be a useful agent for the topical treatment of HPV infections.

The aim of this study was to test the hypotheses that the presence of the entire HPV 16 genome has an effect on the sensitivity of cells to LMB, and that HPV integration influences this sensitivity. We used derivatives of the W12 cell line, which provide a useful model of HPV infection in which the complete genome is present in the natural context as either episomal or integrated forms. The W12 keratinocyte cell lines originate from a low grade squamous intraepithelial lesion (SIL) (4) that contained HPV 16 (6). Parental W12 cells display typical keratinocyte morphology in vitro (4), 
and at early passage contain the entire episomal viral genome, in either the supercoiled or open-circular forms (11). The parental line was clonally selected by Jeon et al (8) to form the episomal and integrated W12 derivatives used in this study.

\section{Materials and methods}

Cell culture. W12 derivatives were a kind gift of Paul Lambert (Wisconsin, USA) and J2 3T3 mouse fibroblast feeder cells were a kind gift of Fiona Watt (London Research Institute, London, UK). The W12 derivatives used were $20850\left(\mathrm{~W}_{12} \mathrm{E}_{50}\right), 20863\left(\mathrm{~W}_{12} \mathrm{E}_{63}\right), 201402\left(\mathrm{~W} 12 \mathrm{I}_{02}\right), 20822$ $\left(\mathrm{W} 12 \mathrm{I}_{22}\right), 20831\left(\mathrm{~W}_{12} \mathrm{I}_{31}\right)$, and $20861\left(\mathrm{~W}_{12} \mathrm{I}_{61}\right)(8)$. Cell culture was performed as described previously with some modifications (8). J2 3 T3 feeder cells were treated with $2 \mu \mathrm{g} / \mathrm{ml}$ mitomycin C (Sigma-Aldrich, MO, USA) for $3 \mathrm{~h}$ then plated at $1-2 \times 10^{5}$ in a T80 tissue culture flask. As the feeders were seeded at constant numbers in all flasks their presence did not affect the results. Feeders were left overnight before addition of $5 \times 10^{5}-1 \times 10^{6} \mathrm{~W} 12$ cells. Epidermal growth factor (Sigma-Aldrich) was added $24 \mathrm{~h}$ after passaging, at $10 \mathrm{ng} / \mathrm{ml}$. J2 3 T3 cells were grown in Dulbecco's Modified Eagles Medium containing 10\% fetal calf serum. Primary human keratinocytes (PHKs) (Promocell, Heidelberg, Germany) expressing HPV 16 E7 and E6/E7 were generated as described previously (3).

Southern blot. DNA was extracted from the W12 derivatives $E_{50}, E_{63}, I_{22}$ and $I_{31}$ using the Hirt method to enrich for low molecular weight DNA (12). Hirt extracted DNA $(2.4 \mu \mathrm{g})$ was digested with either BamHI or HindIII. Undigested and digested W12 DNA was then separated by electrophoresis on a $1 \%$ agarose gel, in combination with digested full length W12 plasmid BamHI purified DNA (pEFHPV-16W12E, GenBank Accession No. AF125673, kind gift of P. Lambert). The gel was depurinated, denatured and neutralised prior to alkaline transfer to Hybond- $\mathrm{N}^{+}$(GE Healthcare, NJ, USA). The ${ }^{32} \mathrm{P}$-labelled probe was prepared using purified W12 plasmid DNA labelled with $\alpha-{ }^{32} \mathrm{P}-\mathrm{dCTP}$ (Perkin-Elmer, MA, USA) by random priming. Prehybridisation $(3 \mathrm{~h})$ and hybridisation (overnight) were carried out at $68^{\circ} \mathrm{C}$ in a Hybaid rolling hybridiser. After hybridisation membranes were washed and exposed to X-ray film $\left(3 / 6\right.$ days at $\left.-80^{\circ} \mathrm{C}\right)$.

Leptomycin B (LMB). LMB was a gift from Novartis (Basel, Switzerland), and was stored as a $10 \mathrm{mM}$ stock solution in absolute ethanol and diluted in appropriate medium before addition to cell cultures.

Monolayer treatment. W12 cells were grown as previously described (8) in a T25 tissue culture flask, with a seeding density of $5 \times 10^{5}$ on $2 \times 10^{4}$ feeder cells. When cultures were semi-confluent, cells were treated with $2 \mathrm{nM} \mathrm{LMB}$ for $40 \mathrm{~h}$. Images were captured using an Olympus CK40 microscope and Olympus SC 40 camera (magnification, x200).

\section{Immunocytochemistry}

Monolayer cells. W12 cells were seeded in Lab-Tek chamber slides (Nalgene, NY, USA) at $2.5 \times 10^{5}$ cells on $1.5 \times 10^{2}$ mitomycin C-treated feeder cells as previously described (8). Semi-confluent cells were treated with $2 \mathrm{nM}$ LMB for $38 \mathrm{~h}$ and immunocytochemistry carried out as described previously (3). The primary antibodies used were: M30 mouse monoclonal (1:100, Roche, Basel) and activated caspase-3 (Asp175) (act-casp-3) rabbit polyclonal (1:100, Cell Signaling, MA, USA). Positively stained cells were counted systematically from one edge of the slide at x500 magnification until the proportion of positive cells reached a plateau, as assessed using the running mean method. Feeder cells appeared morphologically different and were excluded from the counts.

Western blotting. For the 48-h time course experiment, subconfluent cells were treated with $2 \mathrm{nM}$ LMB, with the exception of the untreated zero time point. Treated and untreated time-matched controls were harvested at $0,12,24$, 36 and $48 \mathrm{~h}$. Equivalent amounts of protein were separated in SDS-PAGE gels and transferred to Hybond-P membrane (GE Healthcare). Proteins were detected using primary antibodies to M30 (1:500), and act-casp-3 (1:400). Horseradish peroxidase (HRP)-conjugated goat anti-mouse or anti-rabbit secondary antibody (1:10000, Pierce, IL, USA) was added prior to detection with enhanced chemiluminescence (Immobilon Western, Millipore, MA, USA). Cytokeratin (CK) 18 status was established using a mouse monoclonal antibody [1:500, clone LE65 from the lab of E.B. Lane, University of Dundee, UK (13)]. To confirm equal loading, the membrane was stripped (four 15-min washes in $10 \%$ glacial acetic acid) and re-probed with a $\beta$-actin mouse monoclonal primary antibody (1:5000, Sigma-Aldrich). Secondary antibody and detection were as described above. Quantification was undertaken using Image J software: all measurements given are integrated density in arbitrary units (AU) and normalised against $\beta$-actin.

\section{Results}

The W12 derivatives provide a useful model of HPV 16associated neoplasia with two episomal and four integrated cell lines. The integrated derivatives are also classified by the type of integration. In type 1 integration, single copies of the viral genome integrate causing disruption in the E2 gene (8): this is represented by $\mathrm{I}_{02}, \mathrm{I}_{22}$, and also the cervical carcinomaderived cell line $\mathrm{SiHa}(14) . \mathrm{I}_{31}$ and $\mathrm{I}_{61}$ have undergone type 2 integration, which is also present in the cervical carcinomaderived cell line CaSki; here tandem repeats of full length viral DNA sequences flanked by disrupted viral sequences are integrated into the host DNA (8). Both types of integration have been noted in vivo (8). The presence of episomal genomes in the $\mathrm{E}_{50}$ and $\mathrm{E}_{63}$ derivatives was confirmed by Southern blot analysis (Fig. 1).

LMB induces morphological changes in W12 cells grown in monolayer. When cultured in monolayer the W12 derivatives demonstrated typical keratinocyte morphology and grew in tightly packed cell clusters. After treatment with $2 \mathrm{nM}$ LMB for $40 \mathrm{~h}$, examination by phase contrast microscopy indicated that apoptosis had been induced by LMB in all lines (Fig. 2a). 

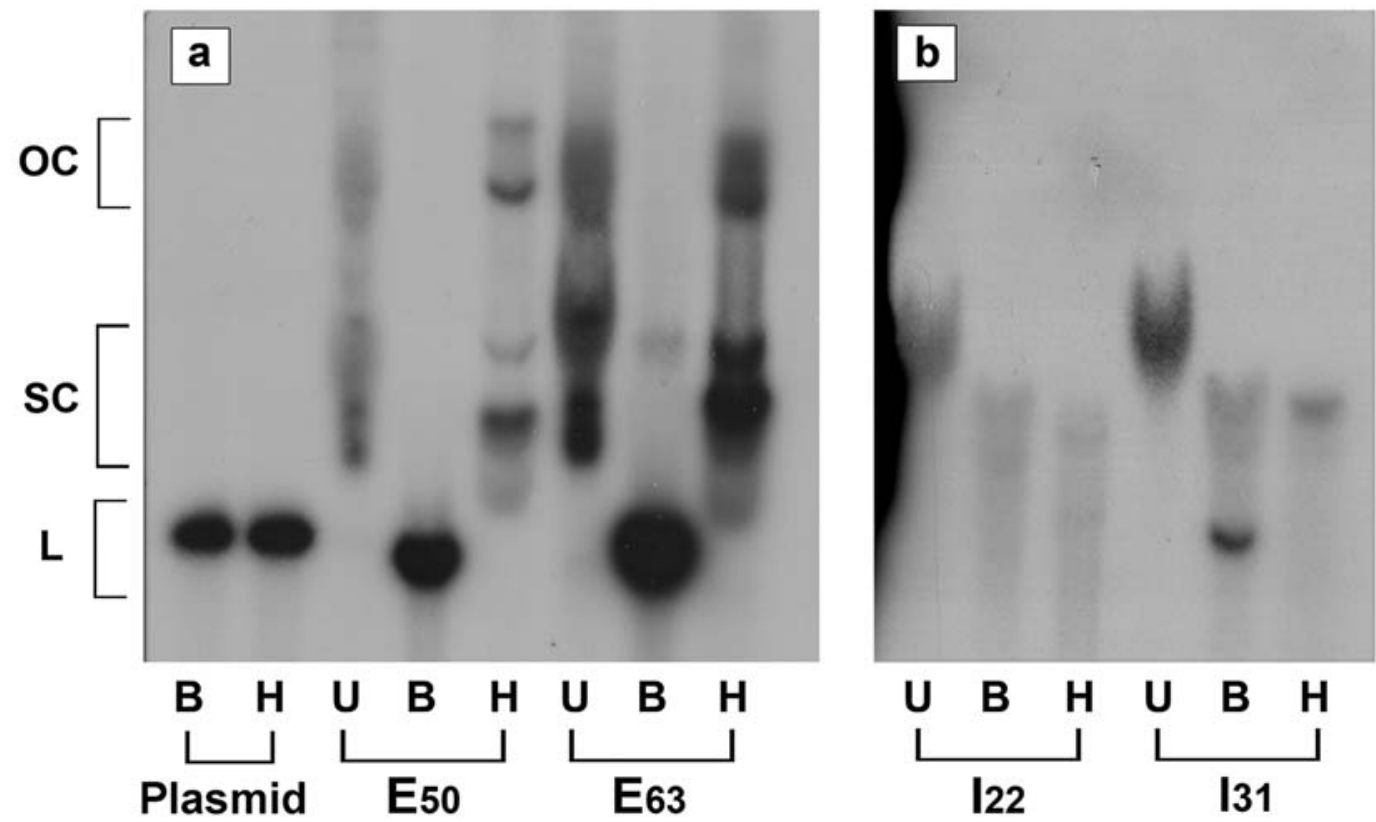

Figure 1. Southern blot analysis of genome status of the W12 derivatives. The genome status of the episomal derivatives $\mathrm{E}_{50}$, $\mathrm{E}_{63}$, and representatives of type 1 $\left(I_{22}\right)$ and type 2 integration $\left(I_{31}\right)$ was analyzed. Images (a) and (b) are taken from the same experiment; however, image (a) shows a 3-day exposure, whilst image (b) shows a 6-day exposure. (a) The presence of high levels of both the open circular (OC) and supercoiled (SC) forms of HPV 16 DNA within the uncut $\mathrm{E}_{50}$ and $\mathrm{E}_{63}$ indicates predominantly episomal viral genomes (23). This was confirmed by the production of a $7.9 \mathrm{~kb}$ band (L) after Bam HI digestion consistent with genome linearization, and the absence of sensitivity to HindIII digestion. (b) As Hirt extracted samples enriched for low molecular weight DNA were used, integrated viral genomes were more difficult to detect and required longer exposure. Full-length HPV genomes were not identified after Bam HI digestion in the $\mathrm{I}_{22}$ line, consistent with type 1 integrated HPV 16. Conversely, full-length genomes were present after Bam HI digestion of $\mathrm{I}_{31}$ DNA, consistent with type 2 integrated HPV 16 as previously noted by Jeon et al (8). HindIII digestion caused a shift in band size in the integrated lines most visibly with the $\mathrm{I}_{31}$ derivative, indicating the presence of host-virus junction fragments.

The addition of LMB to W12 derivatives induces the expression of apoptotic markers. The apparent induction of apoptosis by LMB was verified by examining the expression of two markers of apoptosis, namely act-casp-3 (15) and the M30 neoepitope of CK $18(16,17)$ (Figs. 2b and c).

Activated caspase-3. Immunocytochemistry of the W12 derivatives showed act-casp-3 expression in a small proportion of cells in the absence of LMB, although there was some variation in this expression between the lines (Fig. 2b). Treatment with $2 \mathrm{nM}$ LMB (38 h) caused a marked increase in act-casp-3 expression in all of the W12 derivatives (Fig. 2b). The most marked effect was seen in the $\mathrm{E}_{50}$ cells, with the remaining derivatives all showing lesser responses, particularly the $\mathrm{I}_{02}$ and $\mathrm{I}_{22}$ lines.

This analysis suggested that the apoptotic response to LMB varied between the cell lines. However, as the immunocytochemistry looks only at residual cells, the total cell response in combination with the time-dependence of this effect was investigated in further detail by Western blot analysis over a 48-h time course (Fig. 3). PHKs expressing E7 and E6/E7 genes (3) were compared with the W12 cells containing the whole HPV 16 genome. Western blot analysis revealed a $17 / 19 \mathrm{kDa}$ doublet indicating the presence of the activated form of caspase-3. Very little or no act-casp-3 was detected in the absence of LMB over this time period. In response to $2 \mathrm{nM} \mathrm{LMB}$, increased act-cap-3 production was initiated in all W12 derivatives and the transduced PHKs containing the HPV E7 and E6/E7 genes, as shown in Fig. 3. The transduced lines demonstrated differential patterns of act-casp-3 expression; cells containing E7 alone displayed levels that peaked sharply at $24 \mathrm{~h}$, with cells transduced with E6 and E7 expressing peak levels later at $36 \mathrm{~h}$. The maximum expression of act-casp- 3 was also considerably lower in the E6/E7 cells than in the E7 cells, suggesting a role for E6 in protecting cells from LMB-induced apoptosis. Both the episomal W12 derivatives $\left(\mathrm{E}_{50}\right.$ and $\left.\mathrm{E}_{63}\right)$ displayed a gradual increase in act-casp-3 levels up to $48 \mathrm{~h}$ (Fig. 3). $\mathrm{I}_{22}$ exhibited a lower overall level of act-casp-3; with a sharp rise at $36 \mathrm{~h}$ that continued at $48 \mathrm{~h}$. A similar pattern of expression was observed in the $\mathrm{I}_{61}$ derivative, although the act-casp-3 protein levels were more comparable to the episomal lines. $\mathrm{I}_{02}$ and $\mathrm{I}_{31}$ were notably different in their expression in comparison to the other derivatives. $\mathrm{I}_{31}$ displayed considerably greater expression at $12 \mathrm{~h}$ than the other $\mathrm{W} 12$ derivatives and transduced primary lines; this was followed by a gradual increase to a maximum production at $36 \mathrm{~h}$. The $\mathrm{I}_{02}$ derivative exhibited a pattern similar to the episomal lines; however, expression of act-casp-3 was unusually low.

M30. Act-casp-3 is only one of a number of possible proapoptotic enzymes, which may be expressed transiently; a second marker of apoptosis was therefore used. The M30 antibody binds to a neoepitope of human CK 18, which is produced due to cleavage by a range of activated caspases [including caspase-3,6,7 (16)] early in apoptosis (17). Initially a $40 \mathrm{kDa}$ product is generated, with a $24 \mathrm{kDa}$ protein being produced later in the process, representing a progression in apoptosis (17). Western blot analysis showed that the W12 derivatives all expressed similar levels of CK18 (data not shown). 
(a)
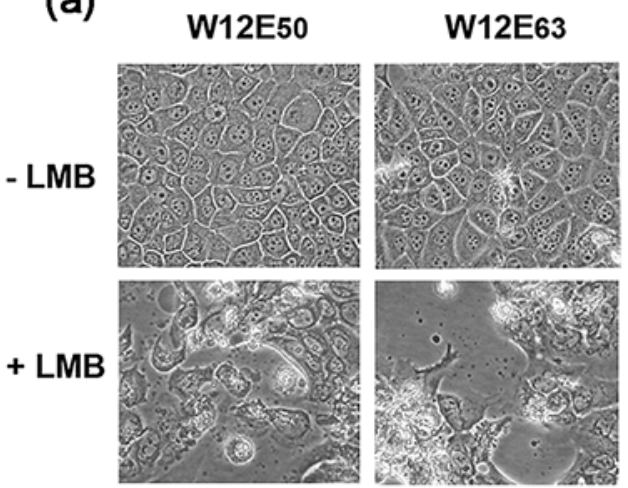

W12102

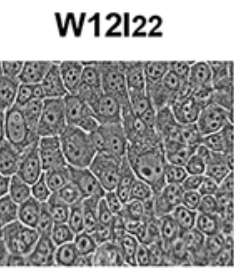

W12131

W12161
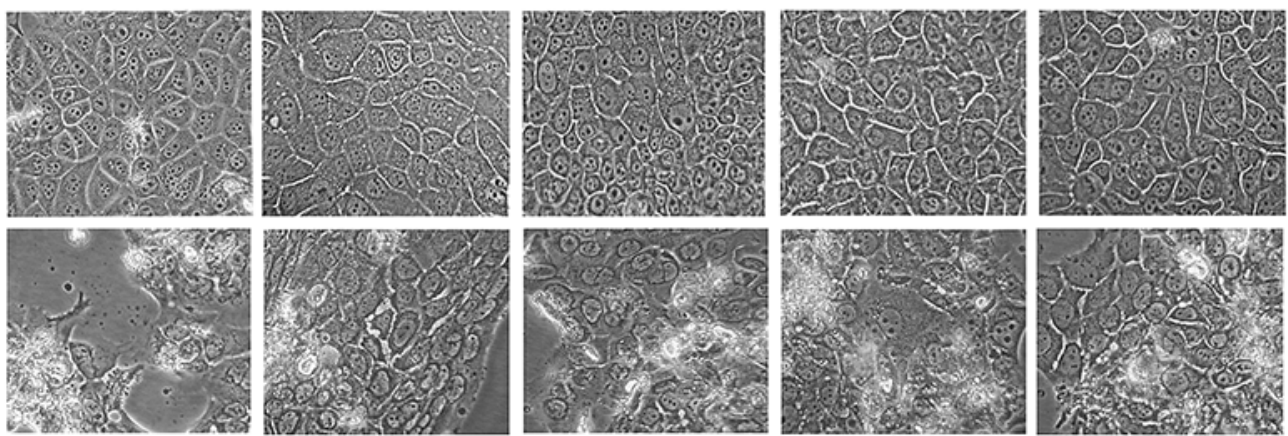

(b)

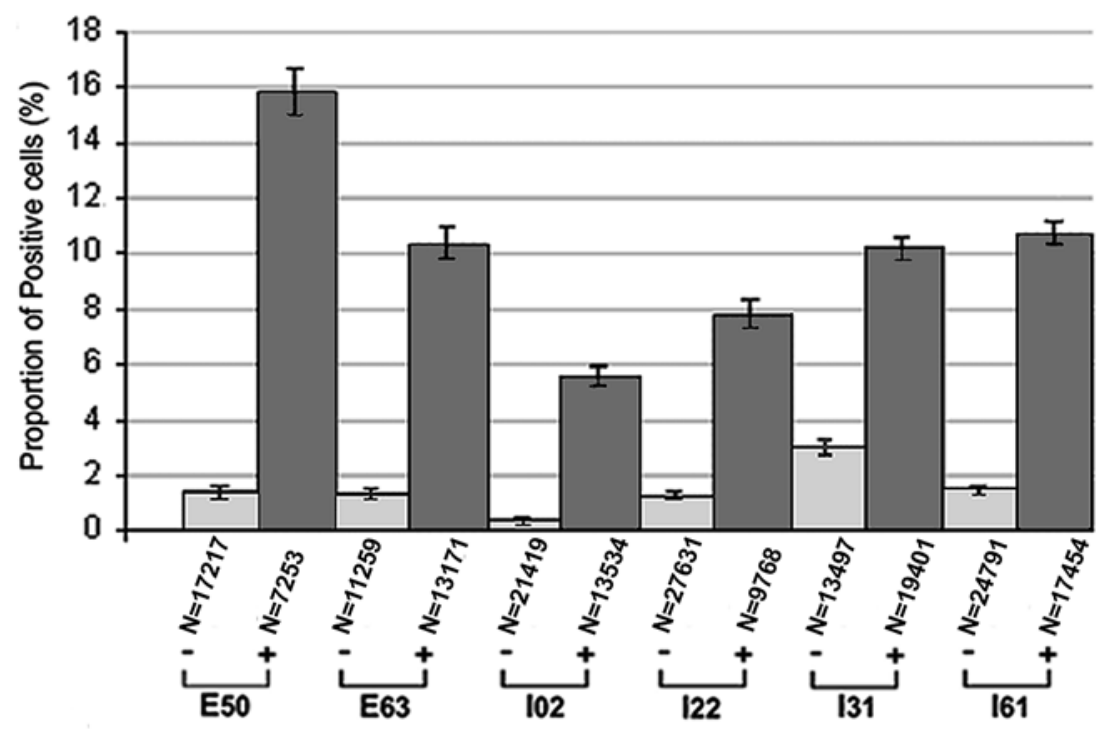

(c)

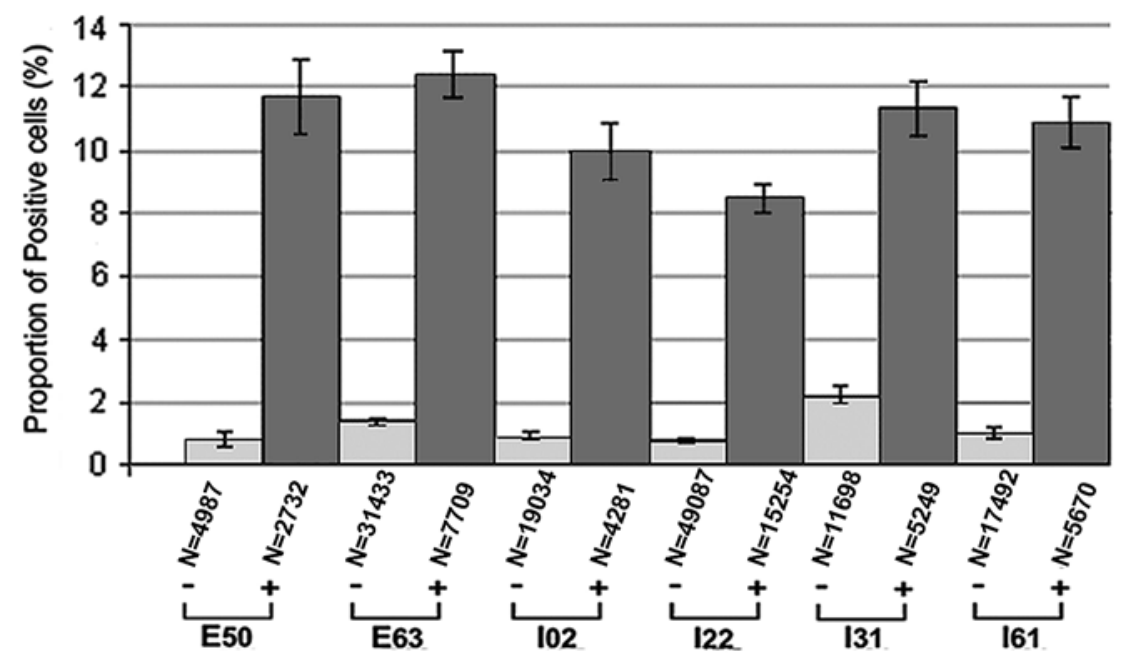

Figure 2. The effect of LMB on monolayer cultures of W12 derivatives. (a) Low power phase contrast images of all the W12 derivatives in monolayer, both untreated (-LMB) and in the presence of $2 \mathrm{nM} \mathrm{LMB} \mathrm{(40} \mathrm{h)} \mathrm{(+LMB).} \mathrm{Magnification,} \mathrm{x200.} \mathrm{The} \mathrm{induction} \mathrm{of} \mathrm{apoptosis} \mathrm{was} \mathrm{assessed} \mathrm{by} \mathrm{immunocytochemistry}$ for act-casp-3 (b) and M30 (c) after 2 nM LMB (38 h). Both treated (+) and untreated (-) cells are shown for each derivative, with $N$ representing the number of counted cells and the error bars representing $\pm 2 \times$ standard error of the proportion (SEP).

Immunocytochemistry revealed low baseline expression of M30 (Fig. 2c) with similar variation in distribution to that of act-casp-3 (Fig. 2b) in all of the W12 derivatives. Considerable induction of M30 occurred in all lines in response to $2 \mathrm{nM}$ LMB (38 h).
As described for act-casp-3, the immunocytochemical results were confirmed by Western blot for the M30 neoepitope (Fig. 4). Analysis of the time course data showed that, in the absence of LMB, expression of the M30 neoepitope could be identified in the W12 derivatives and transduced 
(a)
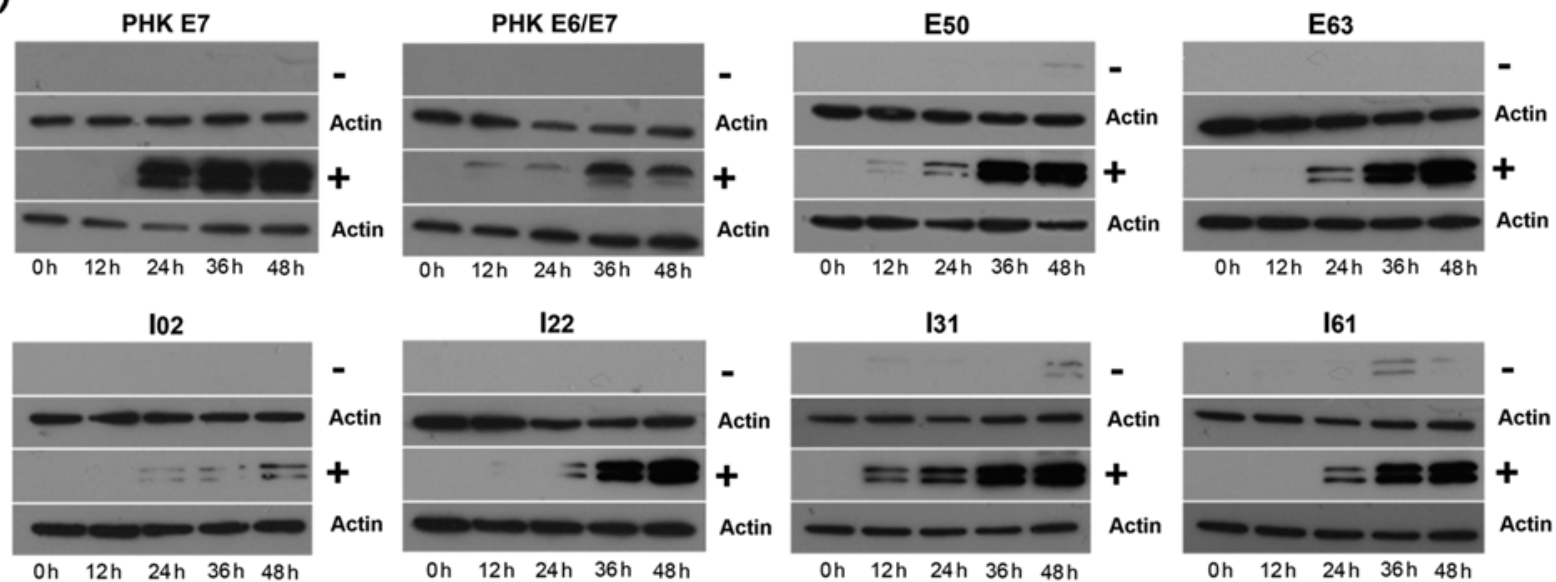

122

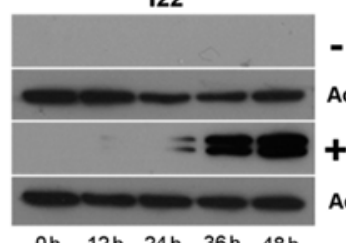

$0 \mathrm{~h} \quad 12 \mathrm{~h} \quad 24 \mathrm{~h} \quad 36 \mathrm{~h} \quad 48 \mathrm{~h}$
131

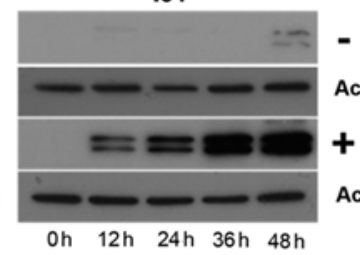

161

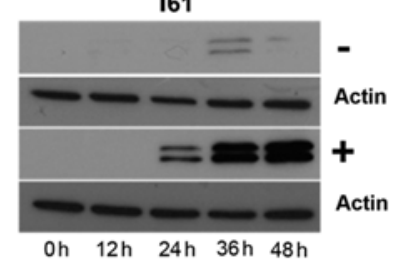

(b)

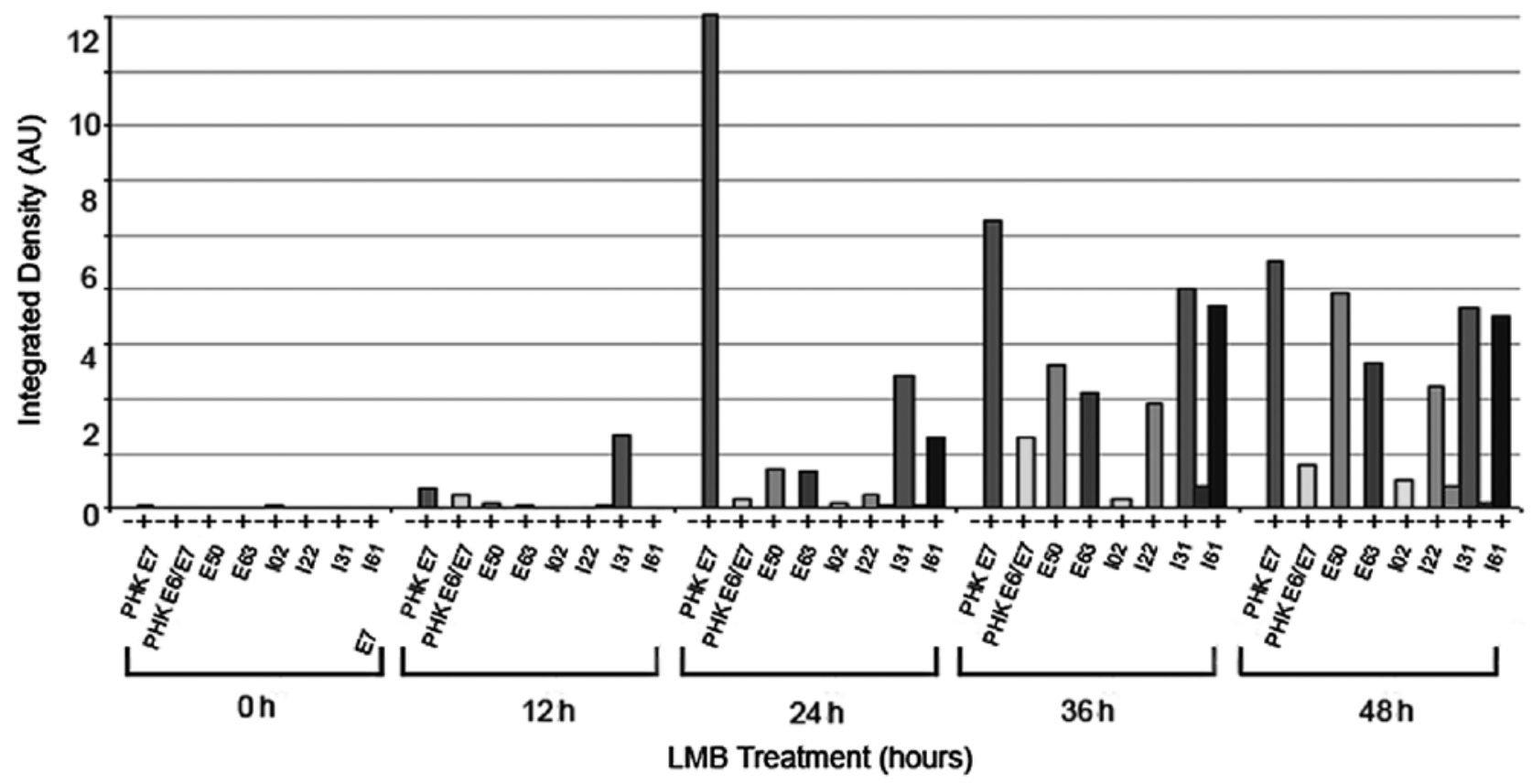

Figure 3. LMB induces the activation of caspase-3 in all of the W12 derivatives and transduced cells over a 48-h time course. (a) Western blot analysis showing the effect of $2 \mathrm{nM}$ LMB on act-casp-3 expression by the PHKs transduced with HPV 16 E7 and E6/E7 and the W12 derivatives over a 48-h time course. Treated and untreated samples were harvested at 12-h intervals with equal loading confirmed by detection of B-actin (Actin); (b) Quantification of blots was undertaken using Image $\mathbf{J}$. All values were normalised against $\beta$-actin for each sample. AU, arbitrary units.

PHKs, although expression was very low in most cases. In response to $2 \mathrm{nM} \mathrm{LMB}$, increased cleavage of CK 18 was induced in all the cell lines (Fig. 4). The transduced PHK lines expressing HPV 16 E7 and E6/E7 each displayed different levels and patterns of M30 protein positivity. PHK E7 exhibited peak levels at the 24-h time point, with a decrease over the subsequent $24 \mathrm{~h}$. The E6/E7 transduced cells displayed a gradual increase in M30 expression over the entire time course. Levels of M30 expression were substantially different between the transduced lines, with the E7 expressing cells displaying over 5 times greater levels than those seen in the E6/E7 line. The episomal W12 derivatives displayed a pattern of M30 expression similar to that seen in the PHKs transduced with E6/E7. Both episomal lines showed a gradual increase in M30 expression up to at least $48 \mathrm{~h}$ of treatment, although E50 displayed marginally greater
M30 expression by $48 \mathrm{~h}$. All the integrated W12 derivatives exhibited a pattern of M30 expression different from that of their episomal counterparts, displaying peak M30 expression after $36 \mathrm{~h}$ of LMB treatment. The integrated W12 cells displayed notable variations in the amounts of M30 produced, with the $I_{02}, I_{22}$ and $I_{31}$ derivatives showing greater levels of M30 than the other cell lines. M30 expression was particularly low for the $\mathrm{I}_{61}$ derivative.

\section{Discussion}

Previous data from our laboratory have shown that leptomycin B induces apoptosis in primary human keratinocytes expressing HPV 16 E7 or E6/E7 genes, but not in normal keratinocytes (3). Moreover, the absence of a notable actcasp-3 or M30 response in wild-type PHKs, despite some 
(a)
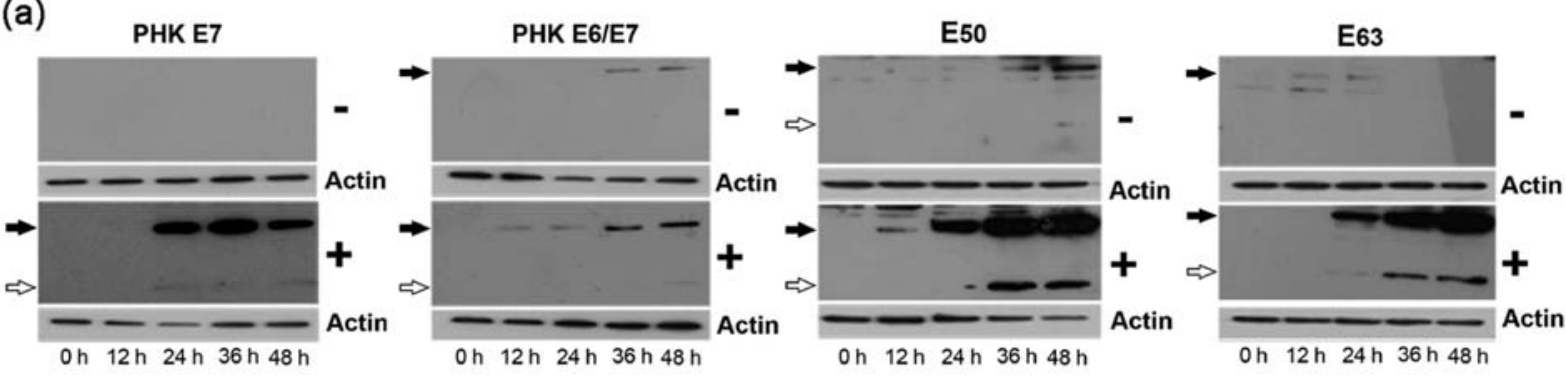

102

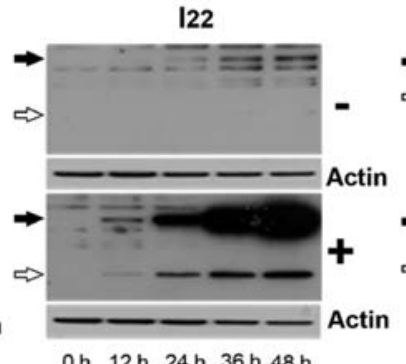

122

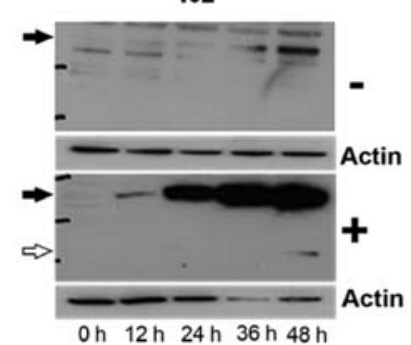

131

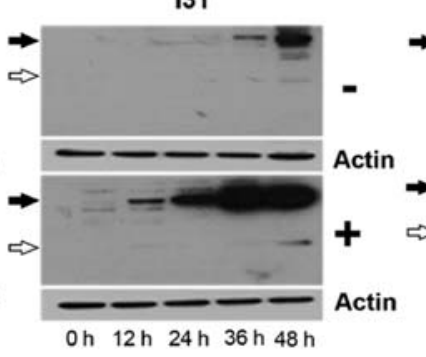

161

(b)

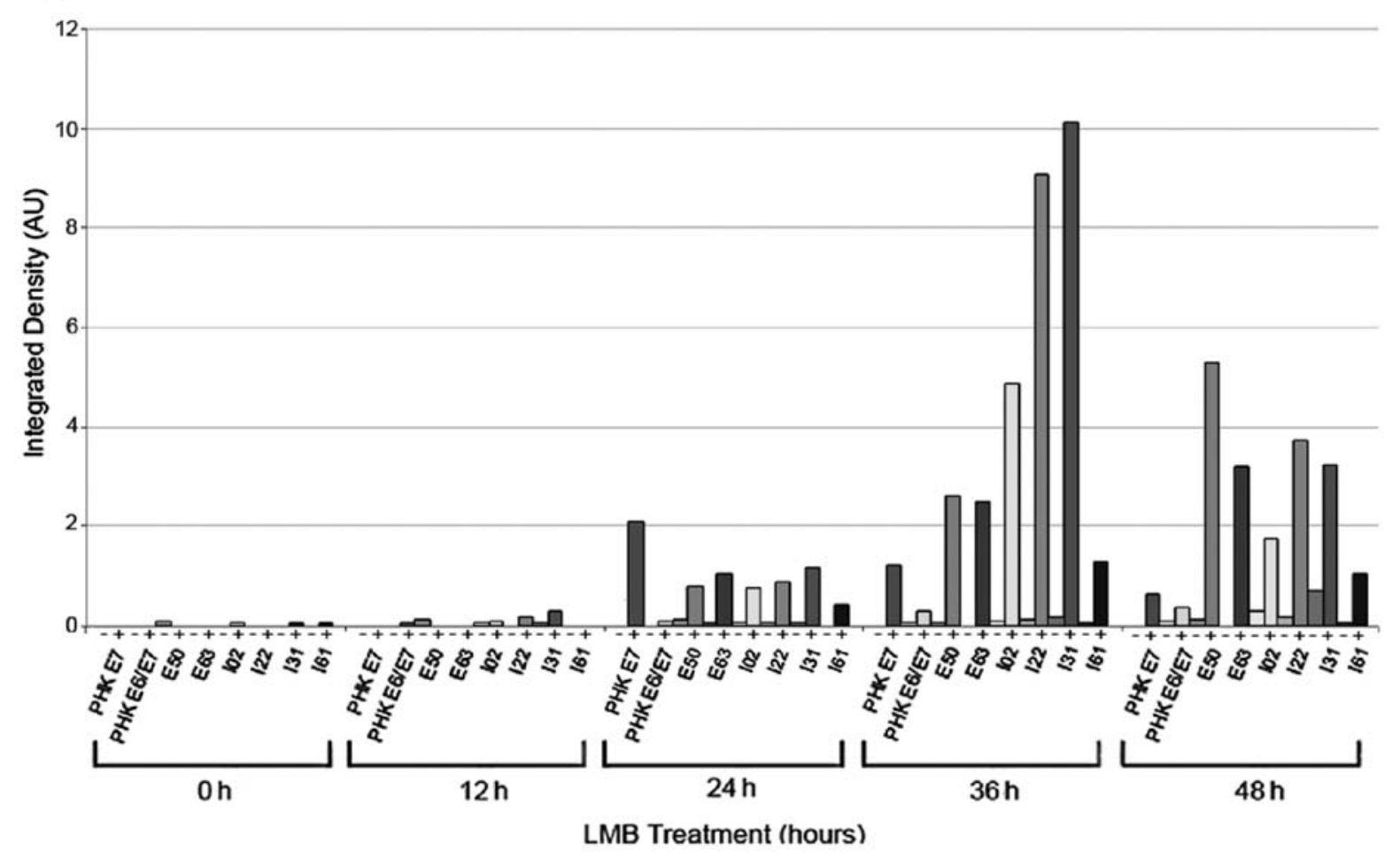

Figure 4. LMB induces the cleavage of cytokeratin 18 to form the M30 neoepitope in all of the W12 derivatives and transduced cells over a 48-h time course. (a) Western blot analysis shows the effect of $2 \mathrm{nM} \mathrm{LMB}$ on total $(40 \mathrm{kDa}$ and $24 \mathrm{kDa}) \mathrm{M} 30$ expression in the W12 derivatives and PHKs transduced with HPV 16 E7 and E6/E7 over a 48-h time course. Where expressed, filled arrows indicate the position of the early $40 \mathrm{kDa}$ form of M30, whilst the empty arrows denote the later $24 \mathrm{kDa}$ cleavage product. Treated and untreated samples were harvested at 12-h intervals with equal loading confirmed by detection of B-actin (Actin); (b) Quantification of blots was undertaken using Image J. All values are shown as total M30 (both early and late forms) and were normalised against $B$-actin for each sample. AU, arbitrary units.

increase in p53 levels (3), suggests that the presence or absence of viral genes influences the cellular response to LMB. In this model the most profound effect was seen in the E7 transduced line, with the presence of E6 having an attenuating effect. Alone, E6 expression induced a weak apoptotic response, although a minimal increase in p53 was observed (3). These results illustrate that, of the two viral oncogenes, E7 is the source of LMB sensitivity, whilst the E6 protein attenuates this response.
The aim of the present study was to assess whether the presence of the complete HPV 16 genome in either episomal or integrated form influences the apoptotic response to LMB. Here we provide evidence that LMB induces a potent increase in apoptosis in derivatives of the W12 cell line containing episomal or integrated HPV 16 genomes. The induction of apoptosis by LMB in these cells was demonstrated not only by changes in morphology, but also by substantial increases in both act-casp-3 and M30 expression in response to treatment. 
The W12 derivatives display considerable inter-derivative variation in the induction of apoptosis, with the status of the viral genome and the clonal nature of the derivatives contributing to the variation in LMB sensitivity. The episomal lines responded differently to $\mathrm{LMB}$, with $\mathrm{E}_{50}$ proving to be more sensitive. As both episomal lines are reported to contain the same viral genome copy number (1000 copies) (8) this is unlikely to be the source of the differential sensitivity. The type 2 integrated $\mathrm{I}_{31}$ and $\mathrm{I}_{61}$ derivatives have a similar medium level act-casp-3 response to LMB; however, $\mathrm{I}_{61}$ displays lower M30 induction than $\mathrm{I}_{31}$. Marker expression in the type 1-integrated derivatives shows fewer similarities. $\mathrm{I}_{02}$ shows a particularly large discrepancy in the expression of each of the apoptotic markers, expressing very low levels of act-casp-3, but high levels of M30; this may be a consequence of its clonal selection from later passage 17 parental W12 cells (8) and may reflect the involvement of alternative apoptotic pathways.

The presence of the HPV 16 genes in specific configurations appears to correlate with temporal expression of the apoptotic markers M30 and act-casp-3. HPV 16 E7transduced cells show the most rapid expression of both apoptotic markers $24 \mathrm{~h}$ after LMB treatment. A dramatic change in sensitivity similar to that shown previously (3) was provoked by the introduction of the HPV 16 E6 gene, delaying apoptosis by at least $12 \mathrm{~h}$; interestingly the presence of E6 also appears to dissociate the expression of the two apoptotic markers. The temporal expression of the apoptotic markers appears to correlate with the integration status of the viral genome in the W12 cell lines. The delay in apoptosis exhibited by the W12 derivatives is reminiscent of that seen in response to the addition of E6 to the transduced cells. The episomal W12 lines show the most prolonged delay in reaching peak levels of act-casp-3 and M30, at least $24 \mathrm{~h}$ after the E7 expressing cells. Both type 1 and type 2 integrated W12 derivatives show a more rapid induction of maximum expression of the apoptotic markers, suggesting an important role for integration in increasing LMB sensitivity. The type 1 integrated derivatives displayed a dissociation in the expression of the markers, suggesting the involvement of other pathways. The type 2 integrated W12s display a coincident peak expression of M30 and act-casp-3 that is also apparent in the PHKs expressing E7. This difference in expression suggests that the type of integration influences the response of these cells to LMB.

The mechanism by which LMB induces apoptosis of the W12 derivatives and the other cell lines appears to be, at least partially, act-casp-3-dependent $(3,18)$. However, in the time course experiment, the W12 derivatives and in particular the type 2 integrated cells, showed a lack of correlation between act-casp-3 and M30 production. LMB-induced act-casp-3 expression in most of the cell lines was either concurrent with or subsequent to M30, suggesting that executioner caspases other than act-casp-3 play an important role in the induction of LMB-induced apoptosis. This is supported by evidence that caspase- 9 plays a specific role in the initiation of apoptosis by LMB treatment of U937 leukaemic cells (18). Particularly high levels of act-casp-3 expression in comparison to M30 were seen after LMB treatment of the E7-transduced keratinocytes and $\mathrm{I}_{61}$ derivative
(Figs. 3 and 4); as both of these lines express higher E7 levels than the other cells studied, (8) the E7 protein may be contributing to this effect. The E6 protein is also likely to play an important role in LMB-induced apoptosis. In addition to the alterations in the pattern of apoptosis, these and previous studies from our laboratory (3) have shown an attenuation of the LMB-induced apoptosis associated with the transduction of E6 into wild-type or E7-transduced PHKs. This effect is likely to be at least in part due to the continued E6-mediated degradation of p53 by nuclear proteasomes (19), but may also represent the interaction of E6 with other components of the apoptotic pathways such as FADD (20), BAK (21) and c-IAP (22).

Our previous data have shown that LMB can induce apoptosis in PHKs expressing HPV oncogenes, but not in wild-type PHKs. This selective effect of LMB suggests that this compound may provide a targeted topical treatment for HPV-associated neoplasia (3). Data from this study suggest that, in a clinical setting, genome status and HPV 16 E6 and E7 oncogene expression may influence the outcome of treatment. For example, lesions containing episomal HPV 16 could require more prolonged or higher dosage treatment with LMB than lesions containing integrated HPV.

In conclusion, the data presented in this study demonstrate that LMB induces apoptosis in keratinocytes containing the whole viral genome in both episomal and integrated states. These data support the investigation of LMB as a therapeutic agent for the treatment of HPV-associated disease.

\section{Acknowledgements}

We thank Dr Paul Lambert for providing the W12 derivatives and Ms. Rachel Lyman for carrying out the transduction of the primary cells. Grant sponsors: University of St Andrews, Maitland Ramsay studentship fund and Cancer Research UK. Leptomycin B was a kind gift from Novartis (Basel).

\section{References}

1. Doorbar J: Molecular biology of human papillomavirus infection and cervical cancer. Clin Sci (Lond) 110: 525-541, 2006.

2. Southern SA and Herrington CS: Molecular events in uterine cervical cancer. Sex Transm Infect 74: 101-109, 1998.

3. Gray LJ, Bjelogrlic P, Appleyard VC, Thompson AM, Jolly CE, Lain $\mathrm{S}$ and Herrington CS: Selective induction of apoptosis by leptomycin B in keratinocytes expressing HPV oncogenes. Int J Cancer 120: 2317-2324, 2007.

4. Stanley MA, Browne HM, Appleby M and Minson AC: Properties of a non-tumorigenic human cervical keratinocyte cell line. Int J Cancer 43: 672-676, 1989.

5. Kalantari M, Blennow E, Hagmar B and Johansson B: Physical state of HPV16 and chromosomal mapping of the integrated form in cervical carcinomas. Diagn Mol Pathol 10: 46-54, 2001.

6. Alazawi W, Pett M, Arch B, Scott L, Freeman T, Stanley MA and Coleman $\mathrm{N}$ : Changes in cervical keratinocyte gene expression associated with integration of human papillomavirus 16. Cancer Res 62: 6959-6965, 2002.

7. Pett $\mathrm{M}$ and Coleman $\mathrm{N}$ : Integration of high-risk human papillomavirus: a key event in cervical carcinogenesis? J Pathol 212: 356-367, 2007.

8. Jeon S, Allen-Hoffmann BL and Lambert PF: Integration of human papillomavirus type 16 into the human genome correlates with a selective growth advantage of cells. J Virol 69: 2989-2997, 1995.

9. Lazo PA: The molecular genetics of cervical carcinoma. Br J Cancer 80: 2008-2018, 1999. 
10. Schiffman M, Herrero R, Desalle R, Hildesheim A, Wacholder S, Cecilia Rodriguez A, Bratti MC, et al: The carcinogenicity of human papillomavirus types reflects viral evolution. Virology 337: 76-84, 2005.

11. Flores ER, Allen-Hoffmann BL, Lee D and Lambert PF: The human papillomavirus type $16 \mathrm{E} 7$ oncogene is required for the productive stage of the viral life cycle. J Virol 74: 6622-6631, 2000.

12. Taylor ER and Morgan IM: A novel technique with enhanced detection and quantitation of HPV-16 E1- and E2-mediated DNA replication. Virology 315: 103-109, 2003.

13. Lane EB: Monoclonal antibodies provide specific intramolecular markers for the study of epithelial tonofilament organization. $\mathrm{J}$ Cell Biol 92: 665-673, 1982.

14. Meissner JD: Nucleotide sequences and further characterization of human papillomavirus DNA present in the CaSki, SiHa and HeLa cervical carcinoma cell lines. J Gen Virol 80: 1725-1733, 1999.

15. Igney FH and Krammer PH: Death and anti-death: tumour resistance to apoptosis. Nat Rev Cancer 2: 277-288, 2002.

16. Caulin C, Salvesen GS and Oshima RG: Caspase cleavage of keratin 18 and reorganization of intermediate filaments during epithelial cell apoptosis. J Cell Biol 138: 1379-1394, 1997.

17. Leers MP, Kolgen W, Bjorklund V, Bergman T, Tribbick G, Persson B, Bjorklund $\mathrm{P}$, et al: Immunocytochemical detection and mapping of a cytokeratin 18 neo-epitope exposed during early apoptosis. J Pathol 187: 567-572, 1999.
18. Jang BC, Paik JH, Jeong HY, Oh HJ, Park JW, Kwon TK, Song DK, et al: Leptomycin B-induced apoptosis is mediated through caspase activation and down-regulation of Mcl-1 and XIAP expression, but not through the generation of ROS in U937 leukemia cells. Biochem Pharmacol 68: 263-274, 2004.

19. Stewart D, Ghosh A and Matlashewski G: Involvement of nuclear export in human papillomavirus type 18 E6-mediated ubiquitination and degradation of p53. J Virol 79: 8773-8783, 2005.

20. Filippova M, Parkhurst L and Duerksen-Hughes PJ: The human papillomavirus $16 \mathrm{E} 6$ protein binds to Fas-associated death domain and protects cells from Fas-triggered apoptosis. J Biol Chem 279: 25729-25744, 2004.

21. Thomas M and Banks L: Inhibition of Bak-induced apoptosis by HPV-18 E6. Oncogene 17: 2943-2954, 1998.

22. Yuan H, Fu F, Zhuo J, Wang W, Nishitani J, An DS, Chen IS, et al: Human papillomavirus type 16 E6 and E7 oncoproteins upregulate c-IAP2 gene expression and confer resistance to apoptosis. Oncogene 24: 5069-5078, 2005.

23. Wettstein FO and Stevens JG: Variable-sized free episomes of Shope papilloma virus DNA are present in all non-virusproducing neoplasms and integrated episomes are detected in some. Proc Natl Acad Sci USA 79: 790-794, 1982. 